\title{
Rundbrief 8.1 (2014)
}

ERNEST W.B. Hess-LÜTTICH

TARTU, DEN 1. MAI 2014

Sehr verehrte Kolleginnen, sehr geehrte Kollegen, liebe Freunde in der GiG,

diesmal schreibe ich Ihnen aus dem estnischen Tartu, unweit der russischen Grenze im hohen Norden des Baltikums. Eine traditionsreiche Universitätsstadt, die den grauen Staub der Sowjet-Ära abgeschüttelt hat und sichtbar erblüht ist, nachdem das Land Mitglied der Europäischen Union und der EuroZone (auch der NATO) geworden ist. Eine Stadt, die vibriert von der Zukunft zugewandter Neugier, von Ideenreichtum und Aufbruchsstimmung, aber auch von Nervosität in bewegter Zeit, von Sorge um das eben Erreichte, auch von Angst vor dem Unberechenbaren in unmittelbarer Nachbarschaft.

Im Postskriptum zu meinem Rundbrief (7.2) zu Silvester 2013 habe ich Ihnen für das von chinesischen Astrologen prophezeite »Jahr der Bewegung « 2014 alles Gute gewünscht und empfohlen, mit ruhiger Hand durch die ja immer zu gewärtigenden Turbulenzen der kommenden Zeit zu steuern. Niemand konnte freilich ahnen, wie turbulent die nächsten Monate werden würden. Aufgrund gravierender politischer Fehler auf allen Seiten spürt Europa unversehens wieder den Eishauch drohender Konfrontationen zwischen Ost und West wie zu längst überwunden geglaubten Zeiten.

Während hektisch entfaltete diplomatische Aktivitäten Schlimmeres zu verhindern suchen, kann der russische Präsident unter ungerührter Verletzung des Völkerrechts binnen weniger Tage mühelos Teile eines Nachbarlandes annektieren. Russland, immerhin noch Mitglied des Europarates, lässt den Rest des westlichen $>$ Bruderlandes $<$, dessen territoriale Integrität es im Budapester Memorandum vor 20 Jahren (am 5. Dezember 1994) zu garantieren noch feierlich geschworen hatte, durch seine kaum getarnten Hilfstruppen destabilisieren und schürt den entfesselten Hass sogenannter pro-russischer Aktivisten, die um sich schießen und einvernehmlich entsandte OSZE-Beobachter als Geiseln nehmen.

Und wie immer spielt die Kirche ihren Part dabei, statt zur Versöhnung aufzurufen, wie es ihres Amtes wäre und Botschaft ihres vorgeblichen Glaubens, hetzen die Popen beider Seiten von den Kanzeln. Die Opfer des Konfliktes erinnern uns mit schmerzlichem Nachdruck daran, wie schmal der Grat ist, auf dem wir balancieren, wie verletzlich der Frieden, wie gefährdet die längst 
für selbstverständlich genommene Sicherheit. Sie erinnern uns daran, dass Freundschaft zwischen Völkern, Respekt gegenüber den Nachbarn, Anerkennung des Anderen, eine mühsam erarbeitete und stets neu zu beglaubigende Kulturleistung ist. Sie erinnern uns daran, dass Verständigung den Versuch voraussetzt, den anderen $\mathrm{zu}$ verstehen, ohne notwendigerweise $\mathrm{zu}$ billigen, wie er handelt oder was er glaubt. Es ist diese Grundeinsicht in die Möglichkeit und Notwendigkeit der approximativen Verständigung über die uns trennenden Grenzen der Kulturen und Weltanschauungen hinweg, die uns eint auch in der Gesellschaft für interkulturelle Germanistik, die uns unverdrossen antreibt, auch in unserem kleinen Gefilde philologischer Forschung und Lehre das Nötige zu tun und das Mögliche zu versuchen, um aus Unwissen erwachsene Fremdheit zu überwinden in der vagen Hoffnung, dass wir dann den uns dadurch vertraut gewordenen Nächsten nicht mehr so schnell liquidieren wollen, nur weil er ein wenig anders und anderer Meinung sein mag als wir selbst. -

Nicht von europäischen Zeitläuften indes wollte ich schreiben - die seltsam ambivalente Stimmung des Ortes forderte ihren Tribut -, sondern mit einem mea culpa wollte ich beginnen: Der längst überfällige Band, der aus unserer schönen Tagung in der alten Kaiserstadt Kyoto noch im nachwirkenden Schatten der Katastrophe von Fukushima erwuchs, dieser Band mit dem Titel Orient im Okzident, Okzident im Orient ist zwar fertig, aber noch nicht als Buch erschienen und versandt. Einen Grund für die Verzögerung hatte ich bereits im letzten Rundbrief angedeutet, die immer leidige Frage der Finanzierung der vom Verlag geforderten Druckkostenzuschüsse.

Bislang war die Gegenleistung der GiG für Ihren Mitgliedsbeitrag im Vergleich $\mathrm{zu}$ anderen Gesellschaften ja konkurrenzlos vorteilhaft. Das war nur möglich durch einen mit der früheren Verlagsleitung persönlich ausgehandelten Vertrag, demzufolge der Verlag für einen Zuschuss von 4.000,00 Euro die gesamte Herstellung (inklusive Layout und teurem Versand) der GiG-Bände gewährleistete. Nun hat die neue Verlagsleitung nachrechnen lassen und mir ihre nicht ganz unerwartet verlustreiche Bilanz für unsere GiG-Reihe vorgelegt. $\mathrm{Zu}$ den bisherigen Bedingungen lasse sich die Reihe schlicht nicht fortsetzen. Um grüne Zahlen zu schreiben, sei ein um 62,5 \% höherer Zuschuss vonnöten als bislang.

Gleichzeitig bangt der DAAD als unser Hauptsponsor um den Erhalt seiner finanziellen Ausstattung im bisherigen Umfang. Zwar ist mit dem neuen Außenminister Frank Walter Steinmeier die Leitung des Auswärtigen Amtes (aus dessen Etat der DAAD finanziert wird) wieder in den Händen eines Politikers, der Auswärtige Kulturpolitik nicht nur als Subvention missversteht, sondern als Investition begreift im Sinne dessen, was ich eingangs schrieb, aber das bedeutet noch nicht unbedingt, dass daraus auch die trivialen Konsequenzen ausreichender Finanzausstattung gezogen würden. Erst im April zeichneten sich die Umrisse des Etats für das laufende Haushaltsjahr ab, sodass entsprechende Anträge auf Unterstützung solange auf Eis lagen.

In fieberhaften Verhandlungen ist es mir nun jedoch gelungen, einerseits beim Verlag ein gewisses Verständnis zu wecken für die Nöte einer gemeinnüt- 
zigen wissenschaftlichen Vereinigung, andererseits beim DAAD die Förderung unserer kommenden Tagung in Limerick im bisherigen Umfang zu erwirken. Kompromisslösungen sind also in Sicht, aber schon dräut das nächste Problem, denn nun gilt es beim Finanzamt für die Erhaltung der Gemeinnützigkeit zu kämpfen, weil die Zahlungsmoral der Mitglieder diese gefährden könnte. Über die Zeit nicht geleistete Beitragszahlungen in namhafter Höhe trotz Inanspruchnahme der Leistungen der GiG sind nicht nur unfair gegenüber den zahlenden Mitgliedern, sondern geben dem Finanzamt (und allfälligen Sponsoren) überdies das Argument in die Hand, erst nach Erreichen des Soll-Saldos über Gemeinnützigkeit (oder Zuschüsse) nachdenken zu müssen.

Daher appelliere ich mit diesem Rundbrief noch einmal an alle Mitglieder, insbesondere die säumigen, die im Vergleich zu den von ihnen empfangenen Leistungen (ein bis zwei umfangreiche Bücher und die Zeitschrift für interkulturelle Germanistik und die subventionierten Treffen bei den alljährlichen Tagungen etc.) allenfalls symbolischen Beiträge von 50,00 Euro (oder gar nur 25, 00 Euro) auch pünktlich jeweils zu Beginn des Kalenderjahres zu entrichten (bzw. auch während der Kongresse, um überhöhte Bankspesen zu vermeiden). Nur dann können die Leistungen erhalten und die absehbaren Beitragserhöhungen verschoben werden.

So will ich nun alles daransetzen, dass noch in diesem Jahr die beiden ausstehenden Bände erscheinen können, die aus den Tagungen in Kyoto (2012) und Johannesburg (2013: Gesellschaften in Bewegung) hervorgegangen sind, und dass die beiden für 2014/15 annoncierten Tagungen wieder mit nennenswerten Unterstützungen durchgeführt werden können. Für die nächste Ende Mai in Limerick stattfindende Tagung ist dies bereits gelungen, und so soll es auch bei der übernächsten in Mumbai wieder sein.

Das attraktive Programm für Limerick zum Thema Begegnungen in Transiträumen / Transitorische Begegnungen hat sich zu einem unerwartet umfangreichen Kongress ausgewachsen, das ich allen Mitgliedern der GiG in der Silvesternacht zugeschickt habe und über dessen neuesten Stand die Homepage jeweils Auskunft gibt: www.ictstudies.eu/internationale-tagung-der-gesellschaft-furinterkulturelle-germanistik-gig.

Ich freue mich darauf, viele von Ihnen bei dieser Gelegenheit in Irland wiederzusehen. Dazu besteht dann sogar noch eine zweite Gelegenheit: im Dezember in Indien. In derselben Nacht vom 31. Dezember 2013 zum 1. Januar 2014 hatte ich den Mitgliedern der GiG auch einen Call for Papers (CfP) zur erwähnten Tagung in Mumbai zugeschickt, der ebenfalls schnell eine erfreuliche Resonanz hatte. So liegen auch für diese Tagung bereits etliche Anmeldungen vor, obwohl der Termin so kurz vor einem für viele Christen wichtigen Fest aus privaten Gründen nicht allen Mitgliedern optimal erschien. Aber die Tagung musste von 2015 auf Ende 2014 vorgezogen werden, weil sie den glanzvollen Abschluss eines ereignisreichen Jahres bilden soll, in dem 100 Jahre Deutschunterricht in Indien, ein Zentennium der Germanistik an den Universitäten von Mumbai und Pune, gebührend gefeiert werden: www.germancentenary. com/events-of-2014. 
Unverständlicherweise sind nicht alle Leser dieser Zeitschrift auch Mitglied der GiG, deshalb sei für diese das Thema und inhaltliche Interesse der geplanten Tagung noch einmal erläutert. Unter dem programmatischen Titel Komparative Ästhetik(en) haben die beiden Organisatorinnen vor Ort, die Kolleginnen Vibha Surana (Professor und Chair of German) und Meher Boot (Assistant Professor) und ich in unserem CfP dazu eingeladen, uns vom 15. bis 21. Dezember 2014 über Ästhetik nicht nur im engeren Sinne als Teilgebiet der Philosophie zu verständigen, sondern als Bestandteil der Produktion, Inszenierung, Rezeption und Funktion von Sprache, Literatur und Medien. In diesem Sinne ist sie auch innerhalb der Germanistik Gegenstand der Literatur- und Medienwissenschaft, der Linguistik und Mediävistik. Und weiter schrieben wir:

Spätestens seit Baumgartens Aesthetica (1750) und Kants Aufruf zur Befreiung des Menschen aus seiner "selbstverschuldeten Unmündigkeit" (1784) lässt sich Ästhetik als ein aufklärerisches Projekt verstehen, das (mit Ernst Cassirer) die "Emanzipation der Sinnlichkeit" zum Ziel hat. Während im 18. Jahrhundert Begriffe der ,Wahrnehmung، (Baumgarten), des 'Schönen، (Kant, Schiller) und der 'Kunst، (Hegel, Schelling) die Ästhetik in Europa prägen, entdeckt das 19. Jahrhundert auch die ,Ästhetik des Hässlichen، (Karl Rosenkranz, Baudelaire, Flaubert u.a.).

Die Ästhetik der Moderne und Postmoderne schließlich ist nicht nur durch Widerstand, Verfremdung, Dissonanz, Gewalt oder Fragmentierung charakterisiert, sondern auch durch Kommunikation (Benjamin), durch die Herrschaft der Technik und den Tod des Subjekts، (Adorno, Foucault, Derrida usw.), aber auch durch die 'Rückkehr des Autorsı (Jannidis, Martinez) und die Expansion des Mediensystems.

Wie , die Ästhetikı, so haben auch andere Begriffe der Kulturwissenschaft (wie ,Literatur oder 'Text`) in den letzten Jahrzehnten eine erhebliche Erweiterung inrer semantischen Extension erfahren. Seit den 1970er Jahren befasst sich die Ästhetik nicht nur mit philosophischen Fragen der Kunst und der Theorie des Schönen, sondern sie wird im weiten Sinne einer allgemeinen Wahrnehmungslehre zur "Begleiterscheinung jeder menschlichen Tätigkeit“ (Joseph Beuys), ja zu einem ubiquitären Faktum des Alltags (cf. Uwe Timm 1993: Erzählen und kein Ende. Versuche zu einer Ästhetik des Alltags; Wolfgang Welsch 1993: Die Aktualität des Ästhetischen; id. 1996: Grenzgänge der Ästhetik).

Prominente Germanisten wie Hartmut Böhme oder Jost Hermand haben mit ihren Schriften zur Ästhetik nach der Postmoderne den Paradigmenwechsel begleitet. Die Entdeckung der Currywurst (Uwe Timm 1993) brachte "Trümmer und Neubeginn, süßlichscharfe Anarchie" mit sich. Germanistik als Kulturwissenschaft und interkulturelle Germanistik entwickeln sich in der fruchtbaren Berührung mit Strömungen in den Kulturwissenschaften fort. Seit langem gibt es komparatistische Studien zu Beziehungen zwischen den Künsten wie Literatur, Malerei, Bildhauerei, Musik, Film oder zwischen Gattungen wie Drama, Prosa, Lyrik, Oper, Fernseh- und Computerspiel, zu intermedialen Relationen zwischen ralten und 'neuen, Medien (Buch und Hörbuch, Literatur und Film, Roman und Drehbuch, Fantasy Genres und Computer Games u.v.m.).

Der ästhetische Erkenntnisgewinn aus komparatistischer Perspektive lässt sich noch erweitern, wenn germanistische Ansätze außerhalb Mitteleuropas in Betracht gezo- 
gen werden wie sie mittlerweile in Afrika, Amerika, Asien und Australien zur Entfaltung gelangen und wenn deren Vielfalt künstlerischen Schaffens in Sprache, Literatur und Medien dazu in Bezug gesetzt wird mit dem Ziel der Entwicklung neuer Wahrnehmungen, Sehweisen, Fragestellungen im Bezirk "Komparativer Ästhetik(en)". Im Einzelnen ermunterten wir zu Beiträgen zu einschlägigen Themen wie zum Beispiel

Sprache, Kunst und komparative Ästhetik,

Stil und Sprache,

Komparative Ästhetik des Intermedialen,

Komparative Ästhetik interkultureller Kompetenz,

Ansätze der Ästhetik im deutschsprachigen Raum im Vergleich,

Ansätze der Ästhetik außerhalb des deutschsprachigen Raums,

Zum Verhältnis ästhetischer Ansätze innerhalb und außerhalb des deutschsprachigen Raums,

Bedingungen, Formen und Perspektiven einer transnationalen Ästhetik.

$\mathrm{Zu}$ diesen oder verwandten Aspekten haben uns trotz knapper Fristsetzungen viele Vorschläge erreicht, die derzeit vom Conference Committee evaluiert werden. Wenn dieses Heft erscheint, werden voraussichtlich alle, die sich angemeldet haben, bereits darüber informiert worden sein, ob ihr Beitrag angenommen wurde. Für den Fall jedoch, dass der (die) eine oder andere Leser(in) unseren CfP nicht erhalten hat, sich aber durch diesen Text animiert fühlt, eine Teilnahme an der Tagung in Erwägung zu ziehen und dafür einen Themenvorschlag einzureichen, so schreibe er oder sie so schnell wie möglich an Vibha Surana (gigmumbai2014@gmail.com; cc: ernest.hess-luettich@germ.unibe.ch). Denn ich schätze das Conference Committee als so flexibel ein, vor allem solche Beiträge, die ästhetische Konzepte aus verschiedenen kulturellen Kontexten und/oder aus linguistischen Bereichen thematisieren, auch nachträglich noch zu berücksichtigen, denn das Programm würde mit Beiträgen zu japanischen, chinesischen, afrikanischen ästhetischen Diskursen ... ja nur noch bunter.

Dieses Programm wird dann so bald wie möglich zusammengestellt und auf der Homepage publiziert: www.germancentenary.com. Dort folge man dem Link zu GiG Mumbai und finde alles Nötige. Leider können Reise- und Übernachtungskosten von den Veranstaltern (oder von der GiG) nicht übernommen werden. Aber wir hoffen wieder auf eine namhafte Unterstützung des DAAD, um Mitgliedern aus DAC-Ländern (www.daad.de/imperia/md/content/entwicklung/dac-liste.pdf) mit einem Zuschuss zu ihren Auslagen die Teilnahme zu erleichtern. Die Tagungsgebühr beträgt 100,00 Euro, schließt aber dafür die Verpflegung während der Tagung bereits ein. Der Homepage ist aber schon jetzt ein Raster der Programmstruktur zu entnehmen, das natürlich nur vorläufig ist:

$\begin{array}{ll}\text { 15.12.2014 } & \text { Anreisetag } \\ \text { 16.12.2014 } & \text { Anmeldung (am Nachmittag) und Eröffnung (im Plenum) } \\ \text { 17.12.2014 } & \text { Tagung (Plenarvorträge und Sektionen) }\end{array}$


18.12 .2014

19.12 .2014

20.12.2014

21.12.2014
Tagung (Plenarvorträge und Sektionen) und Mitgliederversammlung

Tagung (Plenarvorträge und Sektionen) und Abendveranstaltung gemeinsam mit der German Section der Universität Pune: Abschlussfeier zum Jubiläum 100 Jahre Germanistik an der Universität Mumbai

Gemeinsamer Ausflug

Abreise

Das vorläufige Programmraster reserviert am 18. Dezember 2014 Zeit für die Mitgliederversammlung. Gemäß \8 Abs. 2 der Satzung wird dazu fristgerecht (also mindestens zwei Monate vorher) eine gesonderte Einladung ergehen, die voraussichtlich mindestens die folgenden Tagungsordnungspunkte enthalten wird:

$1 \quad$ Begrüßung und Genehmigung der Einladung

2 Bestellung der Schriftführung

3 Genehmigung des Protokolls der Mitgliederversammlung vom 25.September 2010

4 Genehmigung der Tagesordnung

5 Mitteilungen

6 Bericht des Vorstands

7 Finanzbericht der Geschäftsführung

8 Bericht der Kassenprüfer

9 Aussprache

10 Entlastung des bisherigen Vorstands

$11 \quad$ Satzung

12 Mitgliedsbeiträge

13 Bestellung der Wahlleitung

14 Wahl der Kassenprüfer

15 Wahl des neuen Vorstands

16 Varia

Bis dahin ist aber noch Zeit. Vorher sehen wir uns Ende Mai in Limerick. Dann ist die Krise in Europa voraussichtlich noch nicht vorüber; eine Krise, die der deutsche Außenminister im Gespräch mit der deutsch-türkischen Schriftstellerin Meli Kiyak und mit dem in der Schweiz lebenden russischen Schriftsteller Michail Schischkin als »die schwerste in Europa seit dem Ende des Kalten Krieges « bezeichnet und eindringlich warnt: »Wenn wir nicht achtgeben, droht die Rückabwicklung zivilisatorischen Fortschritts, den wir nach der Auflösung des Ost-West-Konfliktes erleben durften«(Die Zeit 19 v. 30. April 2014, S. 40).

Er erinnert in diesem Zusammenhang auch einen der zentralen Auslöser des unvermittelten Gewaltausbruchs im Süden und Osten der Ukraine, als nämlich das Russische als zweite Amtssprache (kurzfristig) verboten werden sollte, was die Russisch sprechenden Ukrainer auf der Krim und im östlichen 
Donbass auf die Barrikaden trieb: »Wir landen bei all diesen Konflikten am Ende immer bei etwas Kulturellem: Man will seine Identität bewahren, seine eigene Sprache sprechen, seine Traditionen leben und seine Gewohnheiten nicht ändern « (ebd.). Eben deshalb ist gerade in globalisierten Zeiten die Pflege des Gesprächs über (vermeintliche) Kulturgrenzen so wichtig: in dem Maße, wie, um ein berühmtes Wort des legendären deutschen Bundespräsidenten Richard von Weizsäcker zu variieren, die Grenzen ihren Charakter des Trennenden verlieren, wird, wie Steinmeier sagt, die »Identitätssuche im Sprachlichen und Traditionalen « stärker. Der daraus abgeleitete Zielkonflikt birgt die Gefahr, die Vision von einer friedlicheren Welt nachhaltig zu trüben. Nichts kann die Berechtigung des Grundanliegens unserer Forschung und Lehre im Bereich der Trans- und Interkulturalität (am Beispiel deutscher Sprache und Literatur) nachdrücklicher belegen als die nüchterne Einsicht in das, was auf dem Spiel steht.

In diesem Sinne wünsche ich allseits eine friedliche, ertragreiche, dialog- und diskursfreudige zweite Jahreshälfte, verbunden mit einem herzlichen Gruß aus Tartu, nicht weit von der Grenze zu Russland. 
